\title{
Joint User Scheduling, and Precoding for Multicast Spectral Efficiency in Multigroup Multicast Systems
}

\author{
Ashok Bandi, Bhavani Shankar Mysore R, Symeon Chatzinotas, and Björn Ottersten \\ Interdisciplinary Centre for Security, Reliability and Trust (SnT), the University of Luxembourg, Luxembourg. \\ Email: \{ashok.bandi, bhavani.shankar, symeon.chatzinotas, bjorn.ottersten\}@uni.lu
}

\begin{abstract}
This paper studies the joint design of user scheduling and precoding for the maximization of spectral efficiency (SE) for a multigroup multicast scenario in multiuser MISO downlink channels. Noticing that the existing definition of SE fails to account for group sizes, a new metric called multicast spectral efficiency (MC-SE) is proposed. In this context, the joint design is considered for the maximization of MC-SE. Firstly, with the help of binary scheduling variables, the joint design problem is formulated as a mixed-integer non-linear programming problem such that it facilitates the joint update of scheduling and precoding variables. Further, useful reformulations are proposed to reveal the hidden difference-of-convex/concave structure of the problem. Thereafter, we propose a convex-concave procedure based iterative algorithm with convergence guarantees to a stationary point. Finally, we compare different aspects namely MC-SE, SE and number of scheduled users through Monte-Carlo simulations.
\end{abstract}

\section{INTRODUCTION}

The unprecedented growth of mobile data traffic led to the adaptation of full reuse of the spectrum and multi-antenna technologies which significantly improved spectral efficiency (SE) [1]. On the other hand, in scenarios like live streaming of popular events, multiple users are interested in the same data. Realizing multicasting such information to all such users results in efficient utilization of the resources, physical layer multigroup multicasting (MGMC) is introduced in [2]. Noticing the significant improvement in resource utilization, multicasting has been adopted into 3GPP standards [3].

Infeasibility of MGMC systems: In real scenarios, each user needs to be served with certain quality-of-service (QoS); failing to meet the QoS leads to retransmission which significantly decreases the SE of the network. The severe inter-group interference (IGI) and/or poor channel gains may limit some users from meeting their QoS [2], [4]. This scenario is referred to as the infeasibility of the MGMC design in the literature [2], [4]. The infeasibility of MGMC is a crucial problem to be dealt with; it is typically addressed by user scheduling (also referred to as admission control in the literature) [5], [6]. Further, precoding impacts IGI [7], [8], thereby influencing the user scheduling.

Multicast spectral efficiency: In this paper, SE maximization is considered as the objective. The existing works on MGMC systems [2], [5], [6], [8], [9], define the SE as the

This work is supported in part by Luxembourgish national fund FNRBRIDGES project PROSAT.

978-1-7281-8895-9/20/\$31.00 (c) 2020 IEEE sum of the minimum rate within each group. However, this definition of SE does not account for the group sizes. However, in the context of user scheduling, group sizes need to be accounted for along with their minimum rates. To comprehend the necessity, omitting the users with lower channel gains leads to lower IGI and improved the minimum rate of each group. Therefore, SE maximization schedules as few users as possible. Noticing the drawbacks of existing SE definition, in this work, a new metric called multicast spectral efficiency (MC-SE) is proposed to account for the group sizes also.

Joint user scheduling and precoding for MGMC systems: In this work, we consider the joint design of user scheduling and precoding for MGMC scenario in a single cell multiuser MISO downlink channels. Similar to most of the works in the literature, we presume that users are already grouped for the MGMC systems according to some criteria [2], [4], [8]. The design of user scheduling and precoding are coupled [7], [8], [10]. Therefore, the optimal performance requires the joint design of user scheduling and precoding [7]; this is compactly referred to as joint design in this paper. To the best of our knowledge, the joint design for the maximization of MC-SE is not considered in the literature. In this context, related works in the literature and contributions/novelty of the paper are summarized in the sequel of this section.

Related works on joint design of scheduling and precoding: The sum-rate maximization (SRM) for MGMC was initially addressed in [11] under the sum power constraint. Later, the joint design of admission control and beamforming for MGMC systems was addressed in [5] for the power minimization problem. The decoupled design of user grouping and precoding without admission control is considered in [8] for satellite systems. The true joint design of scheduling and precoding is initially addressed in [10] for unicast scenario and in [7] for frame-based MGMC scenario in satellite systems. However, the solutions of [7], [10] considered only SE maximization and its extension to MC-SE is not straightforward. The joint design problem is highly complex as it inherits the complications in non-convex maximization and multiplicative nature and binary variables in MC-SE. The following sketches the canvas of the contributions and the specifics are mentioned in the next subsection.

Contributions: To the best of our knowledge, the joint design of user scheduling and precoding for the MGMC system to maximize the MC-SE is not considered in the literature. To this end, the summary of contributions in this 
work as follows:

- To account for the group sizes along with the minimum rate of groups, a new metric called multicast energy efficiency is proposed; this results in a new objective function posing additional challenges.

- With the help of binary scheduling variables, the joint design problem is posed as a mixed-integer non-linear programming (MINLP). Further, through useful reformulations and relaxations, it is transformed as a differenceof-convex/concave (DC) problem.

- Thereafter, a convex-concave procedure (CCP) based on an iterative algorithm is proposed wherein each iterate a convex problem is solved. Further, a simple procedure to obtain a feasible initial point is proposed which ensures the convergence of the proposed algorithms to a stationary point.

- Finally, the performance of the proposed algorithms affecting two design aspects namely scheduled users and SE are numerically evaluated through Monte-Carlo simulations.

The sequel is organized as follows. Section II presents MGMC system. Section III presents the joint design for MC-SE problem, Section IV presents CCP based algorithm. Section V presents simulations and Section VI concludes the work.

Notations: Lower or upper case letters represent scalars, lower case boldface letters represent vectors, and upper case boldface letters represent matrices. $\|\cdot\|$ represents the Euclidean norm, $|\cdot|$ represents the cardinality of a set or the magnitude of a scalar, $(\cdot)^{H}$ represents Hermitian transpose, $(\cdot)^{T}$ represents transpose, and $\mathbb{R}\{\}$ represents real operation, and $\nabla$ represents the gradient.

\section{SYSTEM MODEL}

We consider the downlink scenario of a single cell multiuser multiple input single output (MISO) system with $M$ transmit antennas at the base station (BS). The number of groups is assumed to be equal to the number of transmit antennas i.e., $M$. All the users are equipped with a single antenna receiver and are assumed to grouped into $M$ groups according to some criteria (e.g., Semi-orthogonality based user grouping [8]). Further, to establish the relevance of the design to the real scenarios, a certain QoS requirement (typically depending on the type of service/application) on the group is assumed. A user failing to meet any QoS requirement associated with the group is simply not scheduled in the current slot.

Signal model: User channels are assumed to be constant and perfectly known during the considered transmission. The noise at all users is assumed to be independent and characterized as additive white complex Gaussian with zero mean and variance $\sigma^{2}$. The transmit power at the BS is limited to $P_{T}$ for each transmission and the BS is assumed to transmit independent data to different groups with unit energy. Let $N_{j}$ and $\mathbf{w}_{j} \in \mathbb{C}^{M \times 1}$ be the number of users and precoding vector of group $j$ respectively, and $\mathbf{W}=\left[\mathbf{w}_{1}, \ldots, \mathbf{w}_{M}\right], \mathbf{h}_{i j} \in \mathbb{C}^{M \times 1}$ and $\gamma_{i j}=\frac{\left|\mathbf{h}_{i j}^{H} \mathbf{w}_{j}\right|^{2}}{\sum_{l \neq j}\left|\mathbf{h}_{i j}^{H} \mathbf{w}_{l}\right|^{2}+\sigma^{2}}$ be the downlink channel and the signal-to-interference and noise ratio (SINR) of user $i$ in group $j$.

Multicast Spectral Efficiency (MC-SE): As mentioned in the introduction, in the context of user scheduling and precoding, the standard SE metric needs to be redefined for MGMC systems to account for group sizes. Towards defining MC-SE, let $\eta_{i j}$ be the binary scheduling variable associated with user $i$ in the group $j$. So, $\eta_{i j}=1$ indicates that user $i$ in group $j$ is scheduled and not scheduled otherwise. Finally, letting $\zeta_{j}$ is the minimum $\mathrm{SE}$ of group $j$, MC-SE for the MGMC systems is defined as,

$$
\mathrm{MC}-\mathrm{SE} \triangleq \sum_{j=1}^{M}\left(\sum_{i=1}^{N_{j}} \eta_{i j}\right) \zeta_{j}
$$

$M C$-SE as total received bits/second/Hz: From the physical layer transmission perspective, the spectral efficiency of group $j$ in MGMC systems is same as unicast systems i.e., $\zeta_{j}$. However, in MGMC scenario, the information transmitted to group $j$ is received by $\sum_{i=1}^{N_{j}} \eta_{i j}$ users. Hence, from the network operator perspective, the number of received bits per second per $\mathrm{Hz}(\mathrm{bps} / \mathrm{Hz})$ of group $j$ is $\left(\sum_{i=1}^{N_{j}} \eta_{i j}\right) \zeta_{j}$. Thus, MC-SE can be seen as total number of received bps/Hz.

Similarly, spectral efficiency (SE) is defined as SE = $\sum_{j=1}^{M} \zeta_{j}$ and total scheduled users (TSU) is defined as TSU $=$ $\sum_{j=1}^{M} \sum_{i=1}^{N_{j}} \eta_{i j}$.

\section{PROBLEM FORMULATION AND DC TRANSFORMATION}

In this section, the joint precoder design and user scheduling problem is mathematically formulated to maximize the MC-SE subject to appropriate constraints on the number of scheduled users in each group, power, and QoS constraints. Letting $\epsilon_{j}$ to be the QoS requirement of group $j$, and $\alpha_{i j}$ to be the slack variable associated with user $i$ in group $j$, the joint design problem is formulated as,

$$
\begin{aligned}
& \mathcal{P}_{1}: \max _{\mathbf{W}, \boldsymbol{\zeta}, \boldsymbol{\eta}, \boldsymbol{\alpha}} \sum_{j=1}^{M}\left(\sum_{i=1}^{N_{j}} \eta_{i j}\right) \zeta_{j}, \text { s.t. } \\
& C_{1}: \eta_{i j} \in\{0,1\}, \forall i, \forall j ; \quad C_{2}: \sum_{i=1}^{N_{j}} \eta_{i j} \geq 1, \forall j, \\
& C_{3}: 1+\gamma_{i j} \geq \alpha_{i j}, \forall i, \forall j ; C_{4}: \log \alpha_{i j} \geq \eta_{i j} \zeta_{j}, \forall i, \forall j, \\
& C_{5}: \zeta_{j} \geq \epsilon_{j}, \forall j ; \quad C_{6}: \sum_{i=1}^{M}\left\|\mathbf{w}_{i}\right\|_{2}^{2} \leq P_{T},
\end{aligned}
$$

where $\boldsymbol{\zeta}=\left[\zeta_{1}, \ldots, \zeta_{M}\right], \boldsymbol{\eta}=\left[\boldsymbol{\eta}_{1}, \ldots, \boldsymbol{\eta}_{M}\right], \boldsymbol{\eta}_{i}=$ $\left[\eta_{i 1}, \ldots, \eta_{i N_{j}}\right] \boldsymbol{\alpha}=\left[\boldsymbol{\alpha}_{\boldsymbol{i}}, \ldots, \boldsymbol{\alpha}_{\boldsymbol{M}}\right], \boldsymbol{\alpha}_{\boldsymbol{i}}=\left[\alpha_{i 1}, \ldots, \alpha_{i M}\right]^{T}$, and $P_{T}$ is the total available power.

Remarks:

- Constraints $C_{1}$ and $C_{2}$ in $\mathcal{P}_{2}$ ensures the design to schedule at least one user in each group.

- For the users not scheduled in group $j$, the constraint $C_{4}$ implies $\log \alpha_{i j} \geq 0$ which is satisfied by the definition 
of rate. On the contrary, for all the scheduled users in group $j$, the constraint $C_{4}$ implies $\log \alpha_{i j} \geq \zeta_{j}$. Hence, $\zeta_{j}$ provides a lower bound for the minimum rate of the group with equality at the optimal solution.

- $C_{5}$ and $C_{6}$ are the QoS and power constraint respectively.

Necessity of low-complexity algorithms for joint design: The problem is $\mathcal{P}_{1}$ is combinatorial due to constraints $C_{1}$ and non-convex due to objective and $C_{3}$ and $C_{4}$. Hence, obtaining the optimal solution to $\mathcal{P}_{1}$ requires an exhaustive search-based user scheduling. Additionally, for each scheduling combination, the corresponding precoding problems need to be solved given in $\mathcal{P}_{1}$ which are also non-convex and NP-hard [2]. Therefore, obtaining an optimal solution to the joint design problem is not only NP-hard but also not known. Thus, in the sequel, we focus on developing low-complexity algorithms that are guaranteed to attain a stationary point of $\mathcal{P}_{1}$.

$D C$ transformation with binary constraints: The problem $\mathcal{P}_{1}$ is combinatorial due to constraint $C_{1}$, and non-convex due to constraint $C_{3}, C_{4}$ and the objective. Letting $\mathcal{J}_{i j}\left(\mathbf{W}, \alpha_{i j}\right) \triangleq$ $\frac{\sum_{l=1}^{M}\left|\mathbf{h}_{i}^{H} \mathbf{w}_{l}\right|^{2}+\sigma^{2}}{\alpha_{i j}}$, the DC transformation of $\mathcal{P}_{1}$ subject to binary constraints as,

$$
\mathcal{P}_{2}: \max _{\mathbf{W}, \boldsymbol{\zeta}, \boldsymbol{\eta}, \boldsymbol{\alpha}} \sum_{i=1}^{N} \sum_{j=1}^{M} f\left(\eta_{i j}, \zeta_{j}\right) \triangleq\left(\eta_{i j}+\zeta_{j}\right)^{2}-\eta_{i j}^{2}-\zeta_{j}^{2}
$$

s.t. $C_{1}, C_{2}, C_{5}$ and $C_{6}$ in (2),

$$
\begin{array}{ll}
C_{3}: & \sum_{l \neq i}\left|\mathbf{h}_{i}^{H} \mathbf{w}_{l}\right|^{2}+\sigma^{2} \leq \mathcal{J}_{i j}\left(\mathbf{W}, \alpha_{i j}\right), \forall i, \forall j, \\
C_{4}: & \left(\eta_{i j}+\zeta_{j}\right)^{2}-2 \log \alpha_{i j} \leq \eta_{i j}^{2}+\zeta_{j}^{2}, \forall i, \forall j .
\end{array}
$$

\section{Remark:}

- Constraint $C_{3}$ and $C_{4}$ in $\mathcal{P}_{2}$ are the simple rearrangements that reveal the DC structure in $C_{3}$ and $C_{4}$ in $\mathcal{P}_{1}$ respectively. Hence, $\mathcal{P}_{2}$ is equivalent to $\mathcal{P}_{1}$.

Continuous DC using relaxation and penalization: Ignoring the combinatorial constraints $C_{1}$, the constraint set of $\mathcal{P}_{2}$ involves the difference of convex (DC) problem. So, the stationary points of such DC problems can be efficiently obtained by convex-concave procedure (CCP). With the aim of adopting the $\mathrm{CCP}$ framework, the binary constraints $C_{1}$ in $\mathcal{P}_{2}$ are relaxed to box constraint between 0 and 1 i.e., $[0,1]$ and further penalized to encourage the relaxed problem to include binary $\eta_{i j} \mathrm{~s}$ in the final solutions. Letting $\lambda>0$ be the penalty parameter and $\mathbb{P}($.$) be the penalty function, the$ penalized continuous formulation of $\mathcal{P}_{2}$ is,

$$
\begin{aligned}
& \mathcal{P}_{3}: \max _{\mathbf{W}, \boldsymbol{\zeta}, \boldsymbol{\eta}, \boldsymbol{\alpha}} \sum_{i=1}^{N} \sum_{j=1}^{M}\left(f\left(\eta_{i j}, \zeta_{j}\right)+\lambda_{1} \mathbb{P}\left(\eta_{i j}\right)\right) \\
& \text { s.t. } C_{1}: 0 \leq \eta_{i j} \leq 1, \forall i, \forall j, C_{2} \text { to } C_{6} \text { in (3). }
\end{aligned}
$$

The entropy based penalty function proposed in [10] i.e., $\mathbb{P}\left(\eta_{i}\right) \triangleq \eta_{i} \log \eta_{i}+\left(1-\eta_{i}\right) \log \left(1-\eta_{i}\right)$ is considered in this work as a penalty function. With proposed relaxation and chosen penalty function, the problem $\mathcal{P}_{3}$ involves maximization of
DC objective subject to DC constraints, hence, the $\mathcal{P}_{3}$ is a DC problem of our interest. In order to apply the CCP framework to $\mathcal{P}_{3}$, a feasible initial point (FIP) needs to supplied. However, the constraint $C_{2}$ in $\mathcal{P}_{3}$ limits the choices of FIPs. Hence, for the ease of finding FIPs, constraint $C_{2}$ in $\mathcal{P}_{3}$ is penalized as,

$$
\begin{aligned}
& \mathcal{P}_{4}: \max _{\mathbf{W}, \boldsymbol{\zeta}, \boldsymbol{\eta}, \boldsymbol{\alpha}} \sum_{i=1}^{N} \sum_{j=1}^{M}\left(f\left(\eta_{i j}, \zeta_{j}\right)+\lambda_{1} \mathbb{P}\left(\eta_{i j}\right)\right) \\
&+\Omega \sum_{j=1}^{M}\left(\sum_{i=1}^{N_{j}} \eta_{i j}-1\right) \\
& \text { s.t. } C_{1}: 0 \leq \eta_{i j} \leq 1, \forall i, \forall j, C_{3} \text { to } C_{6} \text { in (4). }
\end{aligned}
$$

Similar to $\lambda$, by choosing the appropriate value of $\Omega$, the scheduling constraint i.e., $C_{2}$ in $\mathcal{P}_{3}$ can be ensured.

\section{A CCP BAsed Joint Design Algorithm}

In this section, a CCP based algorithm is proposed for joint user scheduling and precoding for multicast energy efficiency (JSP-MC-SE) problem (4). CCP is an iterative framework where in each iteration convexifcation and optimization steps are applied to the DC problem $\mathcal{P}_{4}$. The convexification and optimization steps of $\mathcal{P}_{4}$ of JSP-MC-SE at the iteration $k$ is given as,

- Convexification: Let $\left(\mathbf{W}^{k-1}, \boldsymbol{\eta}^{k-1}, \boldsymbol{\zeta}^{k-1}, \boldsymbol{\alpha}^{k-1}\right)$ be the estimates of $(\mathbf{W}, \boldsymbol{\eta}, \boldsymbol{\zeta}, \boldsymbol{\alpha})$ in iteration $k-1$ respectively. In iteration $k$, linearization of $f\left(\eta_{i j}, \zeta_{j}\right)$ without the constant terms and $\mathbb{P}\left(\eta_{i j}\right)$ is given by: $\tilde{\mathbb{P}}^{k}\left(\eta_{i j}\right) \triangleq \eta_{i j} \nabla \mathbb{P}\left(\eta_{i j}^{k-1}\right), f^{k}\left(\eta_{i j}, \zeta_{j}\right) \triangleq$ $2\left(\eta_{i j}^{k-1}+\zeta_{j}^{k-1}\right)\left(\eta_{i j}+\zeta_{j}\right)-\eta_{i j}^{2}-\zeta_{j}^{2}$. Similarly, linearization of right hand side of $C_{3}$ and $C_{4}$ in (4) is given by $\tilde{\mathcal{G}}_{i j}^{k}\left(\eta_{i j}, \zeta_{j}\right) \triangleq-\left[\eta_{i j}^{k-1}\right]^{2}-\left[\zeta_{j}^{k-1}\right]^{2}+2 \eta_{i j}^{k-1} \eta_{i j}+$ $2 \zeta_{j}^{k-1} \zeta_{j}, \tilde{\mathcal{J}}_{i j}^{k}\left(\mathbf{W}, \alpha_{i j}\right) \triangleq \quad-\mathcal{J}_{i j}\left(\mathbf{W}^{k-1}, \alpha_{i j}^{k-1}\right) \quad-$ $\Re\left\{\nabla^{H} \mathcal{J}_{i j}\left(\mathbf{W}^{k-1}, \alpha_{i j}^{k-1}\right)\left[\begin{array}{c}\left\{\mathbf{w}_{l}-\mathbf{w}_{l}^{k-1}\right\}_{l=1}^{M} \\ \alpha_{i j}-\alpha_{i j}^{k-1}\end{array}\right]\right\}$,

where $\quad \nabla \mathcal{J}_{i j}\left(\mathbf{W}^{k-1}, \alpha_{i j}^{k-1}\right)$

$$
\left[\left\{\frac{2 \mathbf{h}_{i} \mathbf{h}_{i}^{H} \mathbf{w}_{l}^{k-1}}{\alpha_{i j}^{k-1}}\right\}_{l=1}^{M},-\frac{\sigma^{2}+\sum_{l=1}^{M}\left|\mathbf{h}_{i}^{H} \mathbf{w}_{l}^{k-1}\right|^{2}}{\alpha_{i j}^{k-1}}\right]^{T} .
$$

- Optimization: Updated $\left(\mathbf{W}^{k}, \boldsymbol{\alpha}^{k}, \boldsymbol{\eta}^{k}, \boldsymbol{\zeta}^{k}\right)$ is obtained by solving the following convex problem,

$$
\begin{array}{r}
\mathcal{P}_{5}: \max _{\mathbf{W}, \boldsymbol{\zeta}, \boldsymbol{\eta}, \boldsymbol{\alpha}} \sum_{i=1}^{N} \sum_{j=1}^{M}\left(f^{k}\left(\eta_{i j}, \zeta_{j}, t\right)+\lambda_{1} \tilde{\mathbb{P}}^{k}\left(\eta_{i j}\right)\right) \\
+\Omega \sum_{j=1}^{M}\left(\sum_{i=1}^{N_{j}} \eta_{i j}-1\right)
\end{array}
$$

$$
\begin{array}{ll}
\text { s.t. } & C_{1}: 0 \leq \eta_{i j} \leq 1, \forall i, \forall j, \\
C_{2}: \sum_{l \neq i}\left|\mathbf{h}_{j}^{H} \mathbf{w}_{l}\right|^{2}+\sigma^{2} \leq \tilde{\mathcal{J}}_{i j}^{k}\left(\mathbf{W}, \alpha_{i j}\right), \forall i, \forall j, \\
C_{3}: \quad\left(\eta_{i j}+\zeta_{j}\right)^{2} \leq 2 \log \alpha_{i j}+\tilde{\mathcal{G}}_{i j}^{k}\left(\eta_{i j}, \zeta_{j}\right), \forall i, \forall j . \\
C_{4}: \quad \zeta_{j} \geq \epsilon_{j}, \forall j ; \quad C_{5}: \sum_{i=1}^{M}\left\|\mathbf{w}_{i}\right\|_{2}^{2} \leq P_{T} .
\end{array}
$$


The proposed CCP based JSP-MC-SE algorithm iteratively solves $\mathcal{P}_{6}$. However, to guarantee its convergence to a stationary point JSP-MC-SE needs to be initialized with a FIP (kindly refer [12]). In the sequel, a simple procedure is proposed to obtain a FIP that promises the convergence to stationary points which yield better performance.

\section{A. Feasible Initial Point}

Since, the quality of the solution depends on the FIP, the harder task of finding a better FIP is considered through the following procedure.

- Step 1: Initialize $\mathbf{W}^{0}$ with complex random values subject to $\left\|\mathbf{W}^{0}\right\|_{2}^{2} \leq P_{T}$ and calculate initial SINRs $\gamma^{0}$.

- Step 2: Choose $\eta_{i j}=\min \left\{1, \frac{\left.\log \left(1+\gamma_{i j}^{0}\right)\right\}}{\epsilon_{j}}\right\}, \forall i, \forall j$.

- Step 3: The above procedure always yields a feasible solution since trivial solution $\boldsymbol{\eta}^{0}=\mathbf{0}$ is also a feasible solution. However, the procedure usually results a better solution than trivial solution.

- Step 4: $\zeta^{0}, \boldsymbol{\alpha}^{0}$ can easily be derived from $\mathbf{W}^{0}$ and $\boldsymbol{\eta}^{0}$.

Remarks:

- Notice that the initial parameters $\mathbf{W}^{0}, \boldsymbol{\zeta}^{0}, \boldsymbol{\eta}^{0}, \boldsymbol{\alpha}^{0}$ are always feasible to (5). Different $\mathbf{W}^{0}$ in step 1 may lead to different FIPs.

- The FIP obtained by this procedure may not be feasible for the original MC-SE problem $\mathcal{P}_{1}$ unless $\mathbf{W}^{0}$ and $\eta^{0}$ become feasible to $\mathcal{P}_{1}$. However, the final solution obtained by JSP-MC-SE with this FIP becomes feasible to $\mathcal{P}_{1}$ since the final solution satisfies all the constraints in $\mathcal{P}_{1}$.

Letting $\mathcal{P}_{5}(k)$ be the objective value of the problem $\mathcal{P}_{5}$ at iteration $k$, the pseudo code of JSP-MC-SE for the joint design problem is given in algorithm 1.

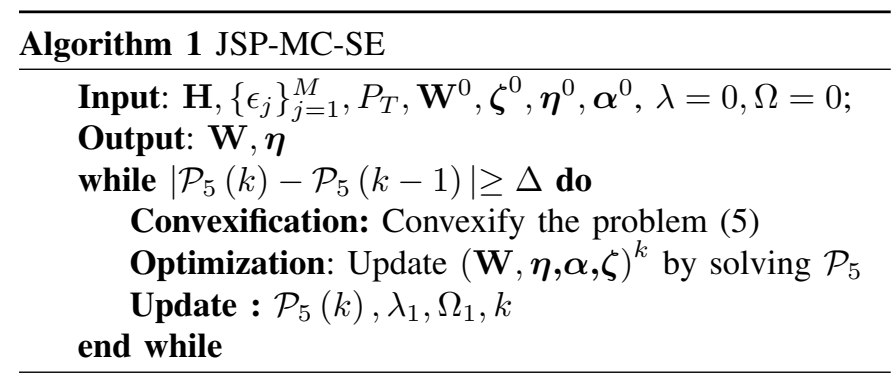

\section{B. Complexity of JSP-MC-SE}

JSP-MC-SE is a CCP based iterative algorithm and its complexity depends on complexity of the convex problem $\mathcal{P}_{5}$. In fact, $\mathcal{P}_{5}$ has $\left(M^{2}+2 \sum_{i=1}^{M} N_{j}+M\right)$ decision variables, $\quad\left(2 \sum_{i=1}^{M} N_{j}+1\right) \quad$ convex constraints and $\left(2 \sum_{i=1}^{M} N_{j}+2 M\right)$ linear constraints. Hence, the computational complexity of $\mathcal{P}_{5}$ is $\mathcal{O}\left(\left(M^{2}+2 \sum_{i=1}^{M} N_{j}+M\right)^{3}\left(4 \sum_{i=1}^{M} N_{j}+2 M+1\right)\right)$ [13]. Commercial software such as CVX can solve $\mathcal{P}_{5}$ efficiently to a large dimension. Besides the complexity per iteration, the overall complexity also depends on the convergence speed of the algorithm. Through simulations, we observe that the JSP-MC-SE converges typically in 15-20 iterations.

Similarly, the above proposed framework can easily be applied to maximize SE and TSU, and their JSP algorithms are simply referred to as JSP-SE and JSP-TSU.

\section{Simulation Results}
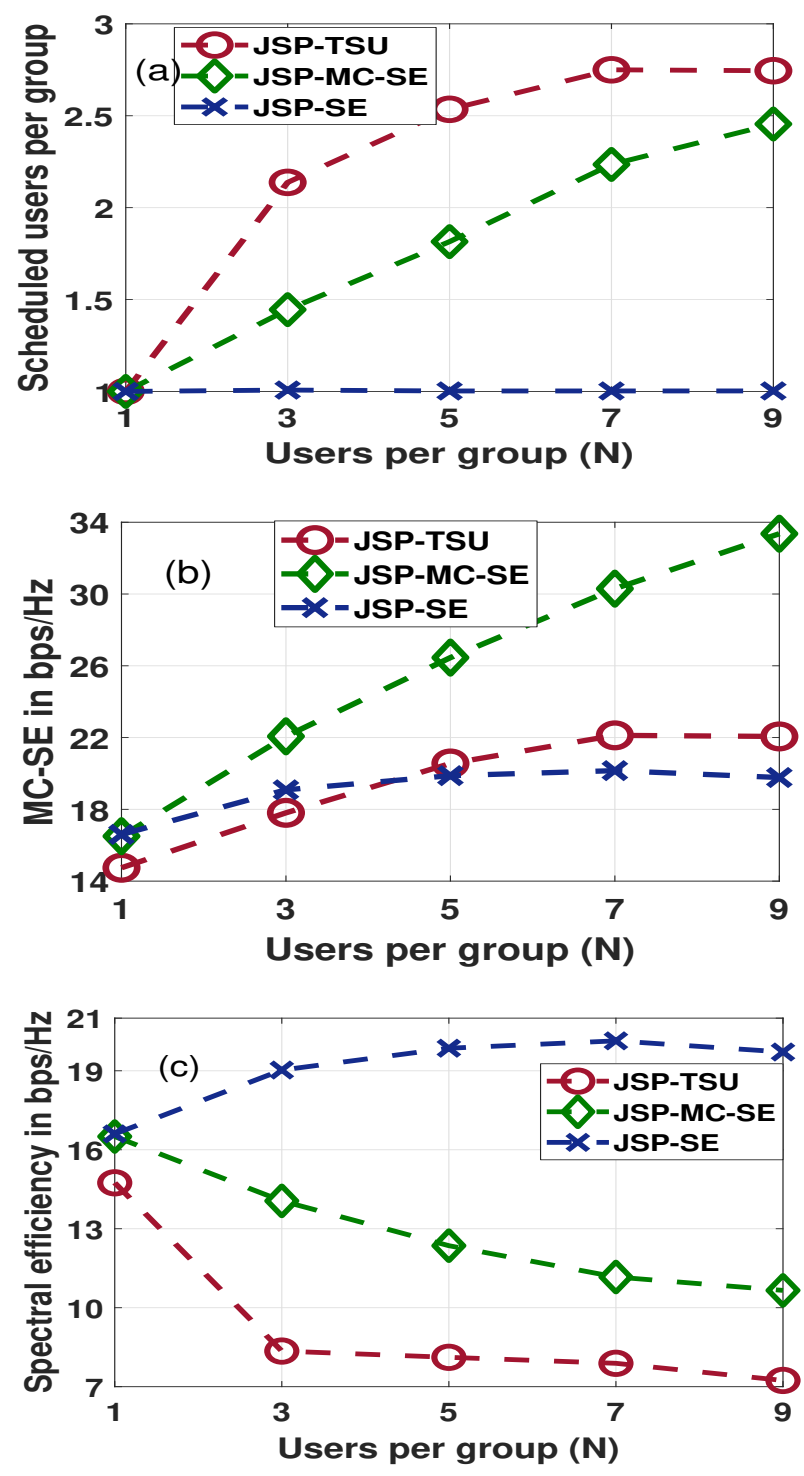

Fig. 1: Comparison of proposed algorithms as a function of $N$ for $M=8$, $\left\{\epsilon_{j}=1 \mathrm{bps}\right\}_{j=1}^{M}$, and $P_{T}=10 \mathrm{~dB}$ (a) number of scheduled UEs per group versus $N$ (b) MC-SE in bps/Hz versus $N$ (c) $\mathrm{SE}$ in bps/Hz versus $N$

In this section, the performance of JSP-MC-SE, JSP-SE and JSP-TSU is evaluated. The system parameters discussed in this paragraph are common for all the figures. The coefficients of the channel matrix, i.e., $\left\{h_{i j}\right\}$ s are drawn from $\mathcal{C N}(0,1)$ and $\sigma^{2}=1$. All the simulation results are averaged over 100 different channel realizations (CRs). Further, $\Omega$ and $\lambda_{1}$ are initialized to 10 and 0.5 respectively and subsequently 
incremented by the factor 1.2 in each iteration. Notice that proposed framework is not sensitive to the initial values of $\Omega$ and $\lambda_{1}$. In figure 1 , the performance of JSP-MC-SE, JSP$\mathrm{SE}$, and JSP-TSU is illustrated as a function of $N_{j}=N, \forall j$ (equally sized groups) varying from 1 to 9 in steps of 2 for $M=8, P_{T}=10 \mathrm{~dB}$ and $\epsilon_{j}=1 \mathrm{bps} / \mathrm{Hz}, \forall j$.

Number scheduled UEs per group versus $N$ : In figure 1a, the number of scheduled UEs per group (NSU) is illustrated as function of $N$. Since JSP-TSU directly maximizes the TSU, it schedules the maximum NSUs compared to JSP-MCSE and JSP-SE. Moreover, due to the low QoS requirement and availability of power, NSU increases with $N$ for JSPTSU. Since the NSU contribute linearly to MC-SE objective, a similar increase in NSU versus $N$ in JSP-MC-SE can be observed in figure 1a. This because an increase in multiuser diversity with $N$ is utilized to schedule a higher number of users by JSP-MC-SE and JSP-TSU which can be observed in figure 1a: this is also achieved by keeping their achieved minimum rate close to the required rates of the groups as illustrated in figure 1c. Hence, the SE by JSP-MC-SE and JSP-TSU decreases with an increase of $N$. However, JSP-MCSE schedules fewer users than JSP-TSU as scheduling these excess users results in a decrease in SE ( as given in figure 1c), thereby, the decrease in MC-SE which can be observed in figure $1 \mathrm{~b}$. On the contrary, the SE objective is not accounting for the number of scheduled users, hence, JSP-SE schedules the lowest number of users i.e. $M=5$. Furthermore, despite the increase in $N$, the number of users scheduled by JSP-SE remains the same. This is because adding a user in a group usually increases IGI and also reduces the minimum rate of the group. Therefore, despite the increase in $N$, JSP-SE schedules only 1 user per group.

$M C$-SE versus $N$ : Recall that MC-SE can be interpreted as the number of received bits per second per $\mathrm{Hz}$ as explained in Section II. It can be seen in figure $1 \mathrm{~b}$, by directly optimizing MC-SE, JSP-MC-SE obtains the highest MC-SE value compared to JSP-SE and JGSP-SUM. The linear increase in MC-SE for $N$ can be observed in JSP-MC-SE and JSP-TSU as the number of scheduled users linearly with $N$ in both the methods. However, as JSP-TSU does not account for SE, it results in poorer MC-SE overall compared to JSP-MC-SE. Unlike JSP-MC-SE and JSP-TSU, MC-SE obtained by JSP-SE is not significant after $N=3$ as it does not gain in scheduled users and the increase in SE is not significant.

$S E$ versus $N$ : In figure $1 \mathrm{c}$, the $\mathrm{SE}$ in $\mathrm{bps} / \mathrm{Hz}$ obtained by proposed algorithms is illustrated as a function of $N$. the JSP-SE objective solely maximizes the SE, hence, it naturally achieves higher SE than JSP-TSU and JSP-MC-SE. Moreover, as $N$ increases the probability of finding $M$ orthogonal users with good channels increases. This leads to a better SE in JSP$\mathrm{SE}$ with an increase of $N$. However, the gains in throughput for JSP-SE diminishes as the gains in multiuser diversity diminish. For $N=1$, JSP-MC-SE and JSP-SE achieve the identical SE as the two metrics are essentially the same. However, JSPTSU achieves lower SE as it does not maximizes SE. On the contrary, for $N>1$, the nature of JSP-MC-SE and JSP-TSU to schedule more users by keeping their achieved rates to the minimum, hence, results in lower SE than JSP-SE.

\section{CONCLUSIONS}

In this paper, the joint user scheduling and precoding is considered for MGMC scenario in MISO downlink channels. To fully leverage the multicast potential, a novel metric called $\mathrm{MC}-\mathrm{SE}$ is considered as a performance metric. Further, with the help of binary scheduling variables and reformulations/relaxations, this problem, first, is formulated as a structured MINLP problem and then transformed into a DC problem. Subsequently, a CCP based algorithm with a simple procedure to obtain feasible initial points is proposed. Finally, the efficacy of the proposed joint techniques is established through simulations and studied their impact on MC-SE, SE, and TSU. This framework can be applied to systems to any number of BS antennas and unequal group sizes. An equal group size scenario with fewer BS antennas is considered in the simulations for representative purposes. Extension of the proposed framework to the imperfect channel state information is left for the future work.

\section{REFERENCES}

[1] ITU-R, "Imt vision framework and oveall objectives of the future development of imt for 2020 and beyond," Tech. Rep., 2015.

[2] E. Karipidis, N. D. Sidiropoulos, and Z. Luo, "Quality of service and max-min fair transmit beamforming to multiple cochannel multicast groups," IEEE Trans. Signal Process., vol. 56, no. 3, pp. 1268-1279, March 2008.

[3] D. Lecompte and F. Gabin, "Evolved multimedia broadcast/multicast service (eMBMS) in LTE-advanced: overview and Rel-11 enhancements," IEEE Commun. Mag., vol. 50, no. 11, pp. 68-74, November 2012.

[4] Z. Xiang, M. Tao, and X. Wang, "Coordinated multicast beamforming in multicell networks," IEEE Trans. Wireless Commun., vol. 12, no. 1, pp. 12-21, January 2013.

[5] E. Matskani, N. D. Sidiropoulos, Z. Luo, and L. Tassiulas, "Efficient batch and adaptive approximation algorithms for joint multicast beamforming and admission control," IEEE Trans. Signal Process., vol. 57, no. 12 , pp. 4882-4894, Dec 2009.

[6] B. Hu, C. Hua, C. Chen, and X. Guan, "User grouping and admission control for multi-group multicast beamforming in mimo systems," Wireless Networks, vol. 24, no. 8, pp. 2851-2866, Nov 2018.

[7] A. Bandi, M. R. Bhavani Shankar, S. Chatzinotas, and B. Ottersten, "Joint scheduling and precoding for frame-based multigroup multicasting in satellite communications," in IEEE Globecom, December 2019.

[8] D. Christopoulos, S. Chatzinotas, and B. Ottersten, "Multicast multigroup precoding and user scheduling for frame-based satellite communications," IEEE Trans. Wireless Commun., vol. 14, no. 9, pp. 4695-4707, Sep. 2015

[9] M. Alodeh, D. Spano, A. Kalantari, C. G. Tsinos, D. Christopoulos, S. Chatzinotas, and B. Ottersten, "Symbol-level and multicast precoding for multiuser multiantenna downlink: A state-of-the-art, classification, and challenges," IEEE Commun. Surveys Tuts., vol. 20, no. 3, pp. 17331757, March 2018.

[10] A. Bandi, B. Shankar M. R, S. Chatzinotas, and B. Ottersten, "A joint solution for scheduling and precoding in multiuser miso downlink channels," IEEE Trans. Wireless Commun., vol. 19, no. 1, pp. 475-490, Jan 2020.

[11] M. Kaliszan, E. Pollakis, and S. Staczak, "Multigroup multicast with application-layer coding: Beamforming for maximum weighted sum rate," in 2012 IEEE WCNC conference, April 2012, pp. 2270-2275.

[12] B. K. Sriperumbudur and G. R. G. Lanckriet, "On the convergence of the concave-convex procedure," in Neural Inf. Proc. Syst., Feb. 2009, pp. 1-9.

[13] P. Gahinet, A. Nemirovski, A. J. Laub, and M. Chilali, LMI Control Toolbox Users Guide. USA: MathWorks, 1995. 